\title{
Chaos in a Relativistic 3-body Self-Gravitating System
}

\author{
F. Burnell ${ }^{1}$ R.B. Mann ${ }^{2}$, \\ Dept. of Physics, University of Waterloo Waterloo, ONT N2L 3G1, Canada \\ T. Ohta ${ }^{3}$ \\ Department of Physics, Miyagi University of Education, Aoba-Aramaki, Sendai 980, Japan \\ PACS numbers: 05.45.Ac, 04.40.b, 04.20.Cv
}

November 19, 2018

\begin{abstract}
We consider the 3-body problem in relativistic lineal (ie $1+1$ dimensional) gravity and obtain an exact expression for its Hamiltonian and equations of motion. While general-relativistic effects yield more tightly-bound orbits of higher frequency compared to their non-relativistic counterparts, as energy increases we find in the equal-mass case no evidence for either global chaos or a breakdown from regular to chaotic motion, despite the high degree of non-linearity in the system. We find numerical evidence for mild chaos and a countably infinite class of non-chaotic orbits, yielding a fractal structure in the outer regions of the Poincare plot.
\end{abstract}

\footnotetext{
${ }^{1}$ email: fburnell@physics.ubc.ca

${ }^{2}$ email: mann@avatar.uwaterloo.ca

${ }^{3}$ email: t-oo1@ipc.miyakyo-u.ac.jp
} 
Self-gravitating $N$-body systems have long been of key importance in studying both stellar dynamics ( of motion, complicated for $N>2$ even in nonrelativistic mechanics due to singularities and evaporation, are essentially intractable for $N>1$ in general relativity because of gravitational radiation. These difficulties are avoided in one-dimensional (or lineal) models of such systems, which describe a system of $N$ parallel mass sheets. Studied by astronomers and physicists for several decades [1], they merit special attention [2, 4], since they not only admit a significantly greater level of computational and analytic analysis but can also be mapped to systems subject to experimental test [3].

We report here on the results of an investigation of the 3-body problem for a relativistic self-gravitating lineal system. Three is the smallest number of particles for which chaotic motions are possible and so such systems are of particular interest. Non-relativistically this system is equivalent to that of a single particle moving in a hexagonal funnel [5] or to that of a uniformly accelerated particle undergoing elastic collisions with a wedge [6]. While its dynamics are well-documented, ours is the first non-perturbative relativistic treatment of this problem. From the canonical formulation of lineal gravity minimally coupled to 3 particles, we obtain an exact expression for the Hamiltonian (valid to all orders in the gravitational coupling $\kappa=8 \pi G / c^{4}$ ), transcendentally expressed as a function of the four independent degrees of freedom. From this we are able to solve for the motion of the system under arbitrary initial conditions and study quasi-periodic motion and chaos in a generalrelativistic self-gravitating system.

For our lineal self-gravitating system we minimally couple $N$ point masses to gravity in 2 spacetime dimensions

$I=\int d^{2} x\left[\frac{\sqrt{-g}}{2 \kappa}\left\{\Psi R+\frac{1}{2} g^{\mu \nu} \nabla_{\mu} \Psi \nabla_{\nu} \Psi+\Lambda\right\}-\sum_{a=1}^{N} m_{a} \int d \tau_{a}\left\{-g_{\mu \nu}(x) \frac{d z_{a}^{\mu}}{d \tau_{a}} \frac{d z_{a}^{\nu}}{d \tau_{a}}\right\}^{1 / 2} \delta^{2}\left(x-z_{a}\left(\tau_{a}\right)\right)\right]$

where $g_{\mu \nu}$ and $g$ are the metric and its determinant, $R$ is the Ricci scalar, $\tau_{a}$ is the proper time of $a$-th particle, and we incorporate a scalar (dilaton) field $\Psi$ since the Einstein action is a topological invariant in 2 spacetime dimensions. The action (1) describes a generally covariant self-gravitating system (without collisional terms, so that the bodies pass through each other) that is a generalization of Jackiw-Teitelboim lineal gravity [7], in which the scalar curvature is equated to a (cosmological) constant $\Lambda$; its equations of motion are

$$
\begin{gathered}
R-\Lambda=\kappa T_{\mu}^{P \mu} \frac{d}{d \tau_{a}}\left\{\frac{d z_{a}^{\nu}}{d \tau_{a}}\right\}+\Gamma_{\alpha \beta}^{\nu}\left(z_{a}\right) \frac{d z_{a}^{\alpha}}{d \tau_{a}} \frac{d z_{a}^{\beta}}{d \tau_{a}}=0 \\
\frac{1}{2} \nabla_{\mu} \Psi \nabla_{\nu} \Psi-g_{\mu \nu}\left(\frac{1}{4} \nabla^{\lambda} \Psi \nabla_{\lambda} \Psi-\nabla^{2} \Psi\right)-\nabla_{\mu} \nabla_{\nu} \Psi=\kappa T_{\mu \nu}^{P}+\frac{\Lambda}{2} g_{\mu \nu}
\end{gathered}
$$

where the stress-energy due to the point masses is

$$
T_{\mu \nu}^{P}=\sum_{a=1}^{N} m_{a} \int d \tau_{a} \frac{1}{\sqrt{-g}} g_{\mu \sigma} g_{\nu \rho} \frac{d z_{a}^{\sigma}}{d \tau_{a}} \frac{d z_{a}^{\rho}}{d \tau_{a}} \delta^{2}\left(x-z_{a}\left(\tau_{a}\right)\right)
$$

and is conserved. Eq. (2) is a closed system of $N+1$ equations for which one can solve for the single metric degree of freedom and the $N$ degrees of freedom of the point masses. The 
evolution of the dilaton field is governed by the evolution of the point-masses via (3). The left-hand side of (3) is divergenceless (consistent with the conservation of $T_{\mu \nu}^{P}$ ), yielding only one independent equation to determine the single degree of freedom of the dilaton.

For general $N$ the canonical formulation of (1) has been studied previously [8] and a number of exact solutions to the 2-body problem have been obtained [9]. Writing the metric as $d s^{2}=-\left(N_{0} d t\right)^{2}+\gamma\left(d x+\frac{N_{1}}{\gamma} d t\right)^{2}$ (with $\int d x \sqrt{\gamma}$ describing proper spatial distance at fixed $t$ ), the action can be canonically reduced to $I=\int d^{2} x\left\{\sum_{a=1}^{N} p_{a} \dot{z}_{a} \delta\left(x-z_{a}\right)-\mathcal{H}\right\}$, where the reduced Hamiltonian is $H=\int d x \mathcal{H}=-\frac{1}{\kappa} \int d x \Psi^{\prime \prime}$, with the overdot and prime denoting $\frac{\partial}{\partial t}$ and $\frac{\partial}{\partial x}$ respectively. The field $\Psi=\Psi\left(x, z_{a}, p_{a}\right)$ is understood to be determined from the constraint equations, which become

$$
\begin{aligned}
\Psi^{\prime \prime}-\frac{\left(\Psi^{\prime}\right)^{2}}{4}+\kappa^{2}\left(\chi^{\prime}\right)^{2}-\frac{\Lambda}{2}+\kappa \sum_{a} \sqrt{p_{a}^{2}+m_{a}^{2}} \delta\left(x-z_{a}\right) & =0 \\
2 \chi^{\prime \prime}+\sum_{a} p_{a} \delta\left(x-z_{a}\right) & =0 .
\end{aligned}
$$

where $\pi=\chi^{\prime}$ and $p_{a}$ are the respective momenta conjugate to $\gamma$ and $z_{a}$. We have chosen the momentum conjugate to $\Psi$ to vanish and $\gamma=1$, fixing the frame of the physical space-time coordinates in a manner analogous to the $(3+1)$-dimensional case [10].

Remarkably eqs. $(5,6)$ can be exactly solved when $N=3$. The solution for $\Psi$ is a sum of exponentials, and for $\chi$ a linear function of $x$ in between each particle. Requiring finiteness of the Hamiltonian at $x= \pm \infty$ and a consistent match of the solutions across the delta-functions at the particle boundaries yields

$$
\begin{aligned}
L_{1} L_{2} L_{3}= & \mathfrak{M}_{12} \mathfrak{M}_{21} L_{3}^{*} e^{\frac{\kappa}{4} s_{12}\left[\left(L_{1}+\mathfrak{M}_{12}\right) z_{13}-\left(L_{2}+\mathfrak{M}_{21}\right) z_{23}\right]} \\
& +\mathfrak{M}_{23} \mathfrak{M}_{32} L_{1}^{*} e^{\frac{\kappa}{4} s_{23}\left[\left(L_{2}+\mathfrak{M}_{23}\right) z_{21}-\left(L_{3}+\mathfrak{M}_{32}\right) z_{31}\right]} \\
& +\mathfrak{M}_{31} \mathfrak{M}_{13} L_{2}^{*} e^{\frac{\kappa}{4} s_{31}\left[\left(L_{3}+\mathfrak{M}_{31}\right) z_{32}-\left(L_{1}+\mathfrak{M}_{13}\right) z_{12}\right]}
\end{aligned}
$$

where $\mathfrak{M}_{i j}=M_{i}-\epsilon p_{i} s_{i j}, M_{i}=\sqrt{p_{i}^{2}+m_{i}^{2}}, L_{i}=H-M_{i}-\epsilon\left(\sum_{j} p_{j} s_{j i}\right), L_{i}^{*}=(1-$ $\left.\prod_{j<k \neq i} s_{i j} s_{i k}\right) M_{i}+L_{i}, z_{i j}=\left(z_{i}-z_{j}\right)$ and $s_{i j}=\operatorname{sgn}\left(z_{i j}\right)$. For simplicity we have set $\Lambda=0$. The discrete constant of integration $\epsilon= \pm 1$ flips sign under time-reversal; it provides a measure of the flow of time of the gravitational field relative to the particle momenta.

Eq. (7) is an exact result and implicitly determines the Hamiltonian $H$ as a function of the two independent coordinate degrees of freedom $(\rho, \lambda)$ and their conjugate momenta, which can be written as

$$
\begin{aligned}
z_{1}-z_{2}=\sqrt{2} \rho & z_{1}+z_{2}-2 z_{3}=\sqrt{6} \lambda \\
p_{1}-p_{2}=\sqrt{2} p_{\rho} & p_{1}+p_{2}-2 p_{3}=\sqrt{6} p_{\lambda}
\end{aligned}
$$

choosing the center of momentum to vanish. The usual relations $\dot{z}_{a}=\frac{\partial H}{\partial p_{a}}$ and $\dot{p}_{a}=-\frac{\partial H}{\partial z_{a}}$ yield the equations of motion, and it is straightforward to show that the Hamiltonian is time-independent when these equations hold.

A post-Newtonian expansion [8] of (7) yields

$$
H=3 m c^{2}+\frac{p_{\rho}^{2}+p_{\lambda}^{2}}{2 m}+\frac{\kappa m^{2}}{\sqrt{8}}\left[|\rho|+\frac{\sqrt{3}}{2}\left(\left|\lambda+\frac{\rho}{\sqrt{3}}\right|+\left|\lambda-\frac{\rho}{\sqrt{3}}\right|\right)\right]-\frac{\left(p_{\rho}^{2}+p_{\lambda}^{2}\right)^{2}}{16 m^{3} c^{2}}+\frac{\kappa c^{2}}{\sqrt{8}}|\rho| p_{\rho}^{2}
$$




$$
\begin{aligned}
& +\frac{\kappa c^{2}}{16 \sqrt{2}}\left[3 \sqrt{3}\left(\left|\lambda+\frac{\rho}{\sqrt{3}}\right|+\left|\lambda-\frac{\rho}{\sqrt{3}}\right|\right)\left(p_{\lambda}^{2}+p_{\rho}^{2}\right)+6\left(\left|\lambda+\frac{\rho}{\sqrt{3}}\right|-\left|\lambda-\frac{\rho}{\sqrt{3}}\right|\right) p_{\rho} p_{\lambda}\right] \\
& +\frac{\kappa^{2} m^{3} c^{6}}{16}\left[\frac{|\rho| \sqrt{3}}{2}\left(\left|\lambda+\frac{\rho}{\sqrt{3}}\right|+\left|\lambda-\frac{\rho}{\sqrt{3}}\right|\right)+\frac{3}{4}\left|\lambda+\frac{\rho}{\sqrt{3}}\right|\left|\lambda-\frac{\rho}{\sqrt{3}}\right|-\frac{3}{4}\left(\lambda^{2}+\rho^{2}\right)(10)\right.
\end{aligned}
$$

in the equal mass case. The first term is the total rest mass and the next two terms are equivalent to the hexagonal-well Hamiltonian of a single particle[5], which we refer to as the hex-particle. We see that to leading order in $c^{-2}$ the hexagon potential is modified to have parabolic edges, as well as a momentum-dependent steepening of its walls. For unequal masses the hexagonal symmetry becomes distorted, with two opposite corners moving inward, changing the relative arc-lengths of the sides. The full potential (obtained from (7) by setting $p_{\rho}=p_{\lambda}=0$ ) has the shape of a hexagonal carafe with convex edges.

The system's interesting dynamics are due to the non-smoothness of the potential along the lines $\rho=0, \rho-\sqrt{3} \lambda=0$, and $\rho+\sqrt{3} \lambda=0$, respectively corresponding to the crossings of particles 1 and 2, 2 and 3, or 1 and 3. We distinguish two distinct types of motion [6]: $A$ motion, corresponding to the same pair of particles crossing twice in a row the hex-particle crossing a single sextant boundary twice in succession), and $B$-motion, in which one particle crosses each of its compatriots in succession (the hex-particle crossing two successive sextant boundaries). We can characterize a given motion by a sequence of letters $A$ and $B$ (called a symbol sequence), with a finite exponent $n$ denoting $n$-repeats and an overbar denoting an infinite repeated sequence.

We numerically solve the equations of motion that follow from (7) in both the exact relativistic $(\mathrm{R})$ system and its non-relativistic $(\mathrm{N})$ limit $($ ie $c \rightarrow \infty)$. We impose absolute and relative error tolerances of $10^{-8}$ for the numerical ODE solvers. This yields numerically stable solutions for the values of $H \leq 6 m c^{2}$ that we study; beyond this the ODE solving algorithm is unstable. We test stability by checking that the energy remains a constant of the motion to within a value no larger than $10^{-6}$ throughout.

For both $\mathrm{N}$ and $\mathrm{R}$ systems we find three principal classes of motion. One class, with sequence $\bar{B}$, describes annulus-shaped orbits encircling the origin in the $\rho-\lambda$ plane. In the second (pretzel) class the hex-particle essentially oscillates back and forth about one of the three bisectors in a combination of $A$ and $B$ motions. Chaotic orbits constitute the third class, where the hex-particle wanders between $A$ - and $B$-motions in an apparently irregular fashion. Such orbits eventually wander into all allowed areas of the $\rho-\lambda$ plane.

Although the orbits of the $\mathrm{N}$ and $\mathrm{R}$ systems realize the same symbol sequences, important qualitative differences exist between them. For a meaningful comparison between each system we set the total energy $H=E_{T}$ and the initial values of $\left(\rho, \lambda, p_{\rho}\right)$ to be the same, setting the initial value of $p_{\lambda}$ to satisfy (7) in each case. As the parameter $\eta=H /\left(3 m c^{2}\right)-1$ grows, we find that $\mathrm{R}$ trajectories have higher frequencies and extend over a smaller region of the $(\rho, \lambda)$ plane than their $\mathrm{N}$ counterparts. Choosing initial conditions so that $A$-motion takes place at $\lambda=0$ we find that $\mathrm{R}$ trajectory patterns narrow with increasing $\eta$ in the small- $\lambda$ region, whereas those for the $\mathrm{N}$ system do not. Figure (1) illustrates a typical example with $\eta=0.5$ for the pretzel case, in which the trajectories for the 3 bodies and their corresponding hex-particle are shown. For each, two of the bodies form a low-amplitude/high-frequency bound state that in turn oscillates at higher amplitude and lower frequency with the third. The 3-body $\mathrm{N}$ oscillations are parabolic in shape whereas there is shoulder-like distortion in 

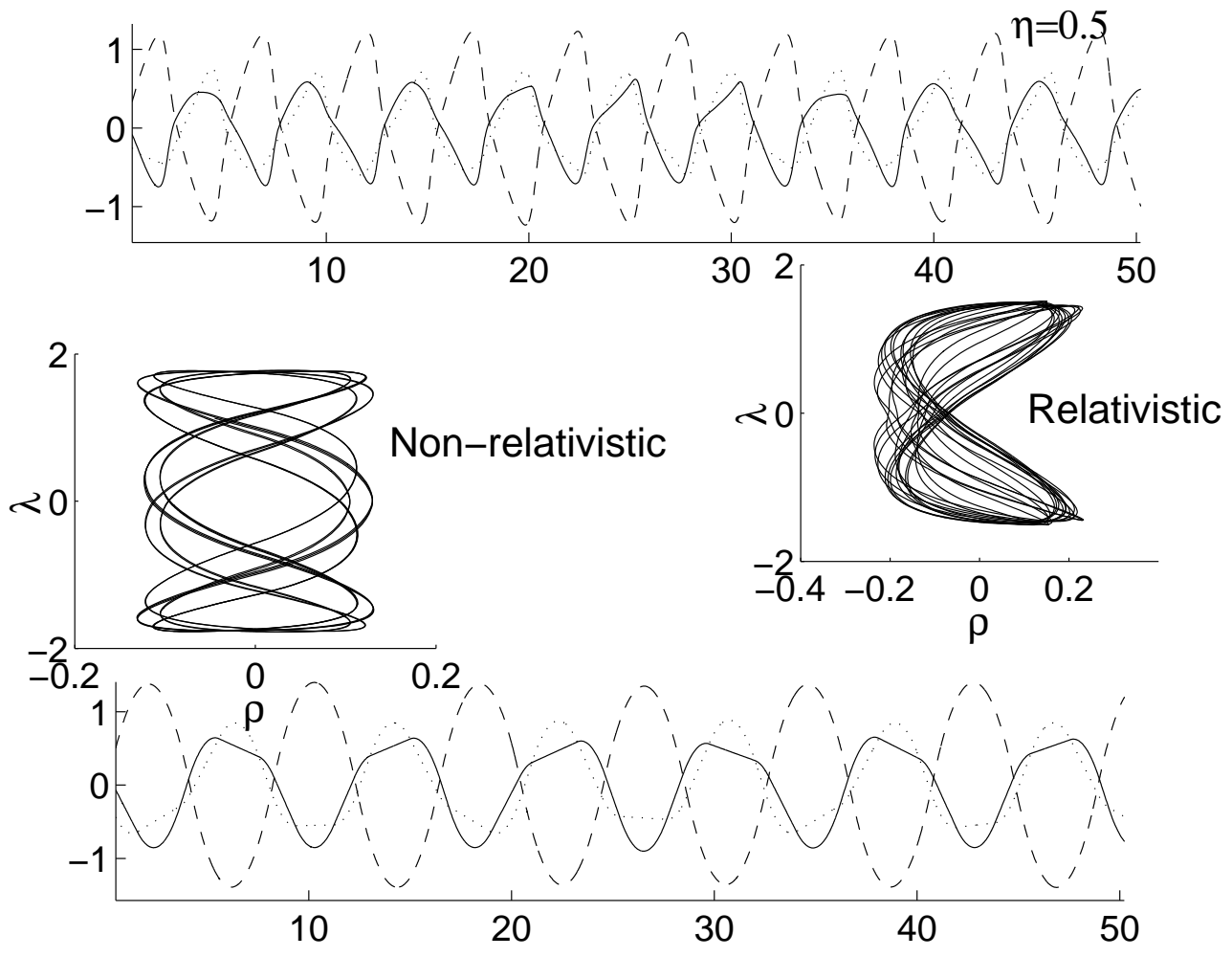

Figure 1: Regular pretzel orbits (run for 120 time steps) of the $\mathrm{R}$ and $\mathrm{N}$ systems with the corresponding 3-particle time evolution (truncated at 50 time steps). The collision sequences differ for these initial conditions and correspond to $A B^{3}(\mathrm{R})$ and $A^{2} B^{3}(\mathrm{~N})$.

the $\mathrm{R}$ system due to the momentum-dependence of the potential (a feature seen previously in the 2-body case [9]) illustrating that under appropriate initial conditions two bodies can tightly and stably bind together in both the $\mathrm{N}$ and $\mathrm{R}$ systems. The hex-trajectories differ substantively, with the $\mathrm{R}$ pattern having $50 \%$ higher frequency, and extending over a slightly smaller range than its $\mathrm{N}$ counterpart. The R-"boomerang" also has an indentation on the left at $\lambda=0$ that is absent for all corresponding $\mathrm{N}$ system boomerangs (not shown).

We probe the global structure of the phase-space using Poincare plots, which we construct by recording the radial $p_{r}$ and angular momentum $j$ each time the hex-particle crosses a hexagonal bisector, plotting their values as a series of dots in the $\left(p_{r}, j^{2}\right)$ plane. Periodic trajectories appear as a finite series of dots, quasi-periodic trajectories as closed loops and chaotic ones as densely filled regions. Our numerical results (see fig. 2 ) for the N system agree with previous studies $[5,6,11]$, which established that a region of phase space exists in which the hex-particle circulates regularly around the origin, bounded by a thin region of chaos outside of which there is a regular structure of periodic and quasi-periodic orbits. Remarkably, we do not observe a breakdown from regular to chaotic motion as $\eta$ increases, despite the high degree of non-linearity in the $\mathrm{R}$ system. The lower regions of the Poincare map clearly display the same pattern of series of circles as occurs in the non-relativistic case. However the Poincare plot for the $\mathrm{R}$ system is no longer symmetric with respect to 


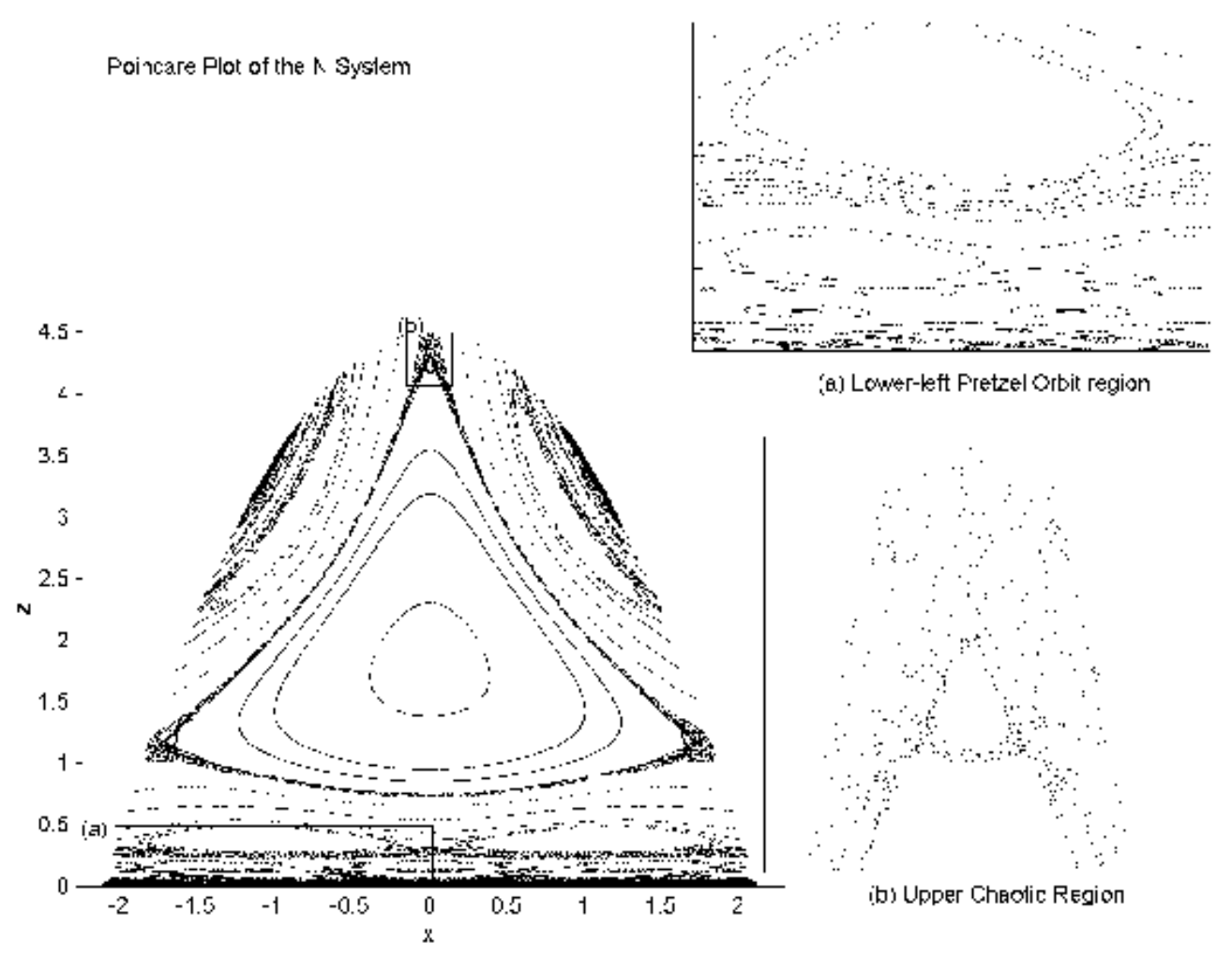

Figure 2: The Poincare plot of the $\mathrm{N}$ system. The squares denote the parts of the plot magnified in the insets.

$p_{r}=0$, but instead is 'squashed' towards the right-hand side (compare fig. 2 to fig.3). This deformation is reminiscent of the situation for two particles, in which the gravitational coupling to the kinetic-energy of the particles causes a distortion of the trajectory from an otherwise symmetric pattern [9]. Since (7) is invariant only under the discrete symmetry $\left(p_{i}, \epsilon\right) \rightarrow\left(-p_{i},-\epsilon\right)$ (and not under $\left.p_{i} \rightarrow-p_{i}\right)$, we obtain for the choice $\epsilon= \pm 1$ a distortion towards the lower right/left-hand side of the plot relative to its $\mathrm{N}$ counterpart.

No sizeable connected areas of chaos are present in either the $\mathrm{R}$ or $\mathrm{N}$ systems, indicating that most trajectories are effectively restricted to move on two-dimensional surfaces in phase space. We find for all $\eta$ within the range investigated that chaotic orbits exist separating the annulus and pretzel regions. We therefore conjecture that the $\mathrm{R}$ system is not integrable, since its $\mathrm{N}$ counterpart is known to be non-integrable [6]. Nonetheless, clearly some underlying feature enforces considerable structure on the phase space, preventing KAM-breakdown to global chaos typical of such Hamiltonian systems [12].

In general pretzel-type orbits display a remarkable richness of dynamics. As the initial angular momentum of the trajectory in question decreases, the number of successive $A$ collisions increases before the hex-particle sweeps around the origin in a $B^{3}$-sequence, corresponding to a 180-degree swing of the hex-particle around the origin . For example $\overline{A B^{3}}$ (the simplest sequence after $\bar{B}$ ) corresponds to a boomerang-type orbit and appears as two circles on the Poincare section. The next simplest sequence is $\overline{A^{2} B^{3}}$, which corre- 


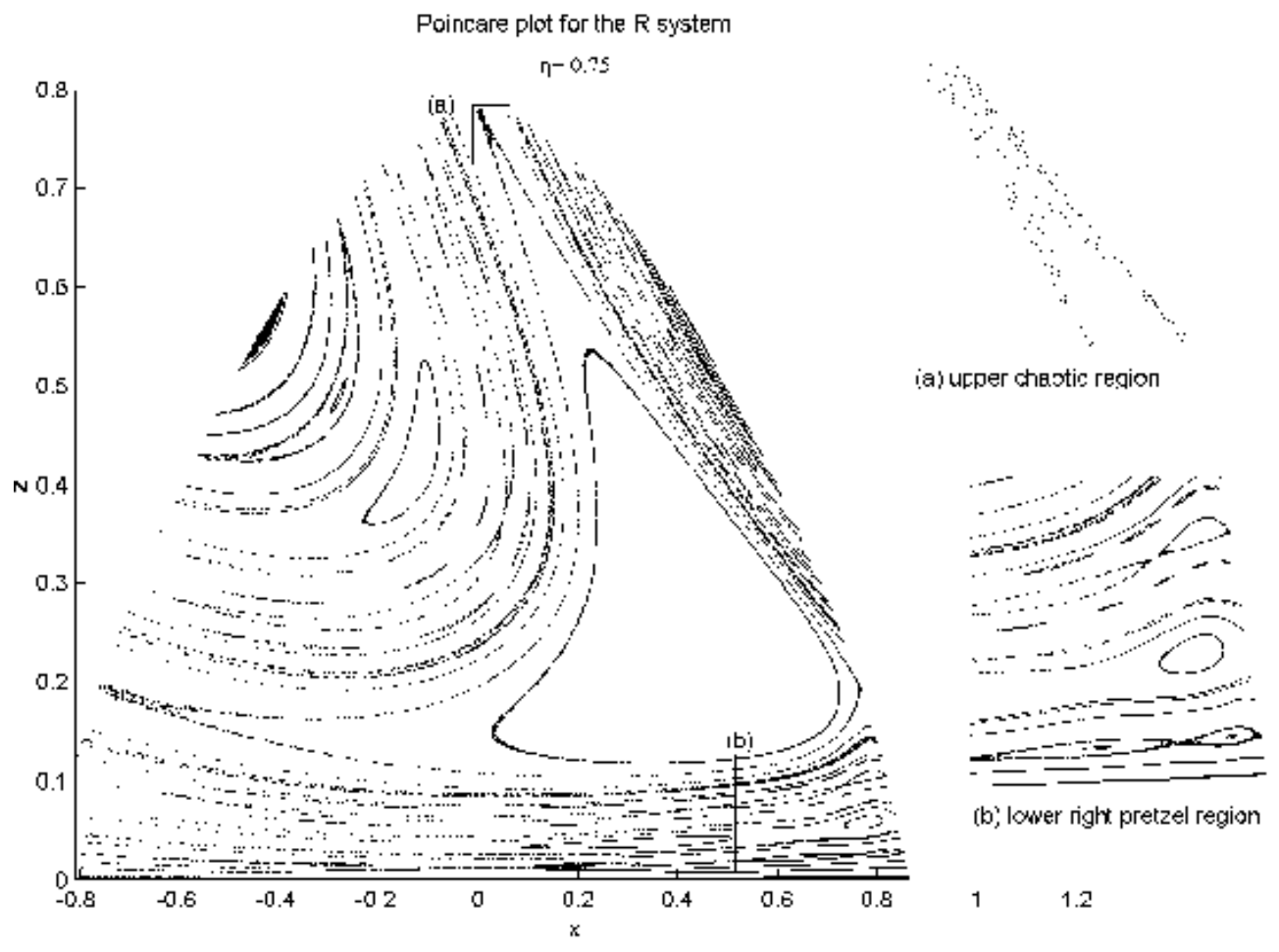

Figure 3: The Poincare plot of the $\mathrm{R}$ system at $\eta=0.75$ and $\epsilon=+1$. The upper right inset provides a close-up of the chaotic region at the top of the diagram; it is now considerably narrower than for lower values of $\eta$. The lower-right inset is a close-up of the structure in a pretzel region in the lower right of the diagram. 
sponds to a bow-tie like orbit, and generates three slightly smaller circles on the Poincare section. For each of these patterns a family of orbits exists corresponding to different widths of the 'bands' of phase space that the trajectory covers, and correspondingly different radii of circles in the Poincare section. Between these regions, the orbits' sequences are mixtures of $A B^{3}$ and $A^{2} B^{3}$. This reasoning can be extended to more general $A^{n} B^{3 m}$-motions. We conjecture that the only allowed non-chaotic orbits - relativistic and non-relativistic - are of the form $\prod_{n, m, j}\left(A^{n} B^{3 m}\right)^{l_{j}}$ with $n, m$ finite, corresponding to increasingly complex weaving patterns.

If the set of integers $l_{j}$ is finite, then the sequence is regular, leaving bands of phase space untravelled, and appearing as a series of closed crescents or ellipsoids on the Poincare section. If, however, the sequence of integers $l_{j}$ never repeats itself, then the trajectory will fill the available phase space densely, appearing as a wavy line on the surface of section. We conjecture that there is a $1-1$ correspondence between rational numbers and periodic orbits in this region of phase space, both non-relativistically and relativistically. This would give the lower section of the Poincare plot a fractal structure as the patterns of circles, ellipses and lines is repeated on arbitrarily small scales as the hex-particle's angular momentum approaches zero.

The 3-body relativistic system provides a new theoretical laboratory for studying the interplay between relativity and chaos. While the dynamical structure of the relativisitc system bears some resemblance to well-known features of its non-relativistic counterpart (such as the self-similar structure of the pretzel class and the lack of energy dependence of the orbit topology), significant differences arise in the shape and frequency of the orbits. A full study of the large- $\eta$ regime should provide us with interesting new information to this end.

This work was supported by the Natural Sciences and Engineering Research Council of Canada.

\section{References}

[1] See G. Rybicki, Astrophys. Space. Sci 14 (1971) 56 and references therein.

[2] See B.N. Miller and P. Youngkins Phys. Rev. Lett. 814794 (1998); K.R. Yawn and B.N. Miller, Phys. Rev. Lett. 793561 (1997) and references therein.

[3] V.Millner, J.L.Hanssen, W.C. Campbell, and M.G. Raizen, Phys. Rev. Lett. 86, 1514 (2001).

[4] R.B. Mann and P. Chak, Phys. Rev. E65 026128 (2002).

[5] D. Butka, G. Karl and B. Nickel, Can J. Phys 78, 449 (2000).

[6] H.E. Lehtihet and B.N. Miller, Physica 21D, 93 (1987).

[7] R. Jackiw, Nucl. Phys. B 252, 343 (1985); C. Teitelboim, Phys. Lett. B 126, 41 (1983).

[8] T. Ohta and R.B. Mann, Class. Quant. Grav. 132585 (1996) (gr-qc/9605004). 
[9] R.B. Mann and T. Ohta, Phys. Rev. D57 4723 (1997); Class. Quant. Grav. 141259 (1997) ; R.B. Mann, D. Robbins and T. Ohta, Phys. Rev. Lett. 823738 (1999); ibid Phys. Rev. D60 104048 (1999); R.B. Mann, D. Robbins, T. Ohta, M.R. Trott, Nucl. Phys.B590, 367 (2000) .

[10] R. Arnowitt, S. Deser and C.W. Misner Gravitation: An Introduction to Current Research, Chap.7 (New York: Wiley 1962); R. Arnowitt, S. Deser and C.W. Misner J. Math. Phys.1 434 (1960); T. Kimura, Prog. Theor. Phys.26 157 (1961); T. Ohta and T. Kimura, Classical and Quantum Gravity, Chap.6 (in Japanese) (Tokyo: MacGrawHill 1989); T. Ohta, H. Okamura, T. Kimura and K. Hiida Prog. Theor. Phys51 1598 (1974).

[11] B.N. Miller and H. Reidl Phys Rev A6, 1554 (1992) .

[12] V.I. Arnold and A. Avez, Ergodic Problems of Classical Mechanics (Springer: New York, 1968). 